\title{
Study on the Spatial - Temporal Variability of Soil Nutrients during Winter Wheat Growth Season
}

\author{
Bei Cui ${ }^{1,2}$, Wude Yang ${ }^{1, *}$, Meichen Feng ${ }^{1}$, Wenjiang Huang ${ }^{2}$, and Xiaoyu Song ${ }^{2, *}$ \\ ${ }^{1}$ College of Agronomy, Shanxi Agricultural University, Taigu 030801, China \\ ${ }^{2}$ Beijing Research Center for Information Technology in Agriculture, Beijing 100097, China \\ \{cuibei1214, fmc101, yellowstar0618\} @163.com, \\ sxauywd@126.com, songxy@nercita.org.cn
}

\begin{abstract}
The object of the study is to analyze the spatial-temporal variability of soil nutrients in the winter wheat growing season, and try to reveal the dynamic change of soil nutrients in winter wheat field. Measurements of soil nitrate nitrogen (NN), total nitrogen (TN) and available potassium (AK) in wheat field were taken at 132 sample points in April and June, 2006. Classical statistical method and geostatistics method were used in this study. The classical statistical analysis results indicate the average value of $\mathrm{NN}$ content decreased obviously from April to June, and the value of AK decreased slightly. But there were no change for the mean of $\mathrm{TN}$ content from April to June. All soil nutrient both in April and June were medium-degree variations. From the spatial distribution maps of soil variables, soil NN changed obviously on distribution between April and June. However, soil TN and AK had the similar spatial distribution that high in the northeast part and low in the southwest of the study field. In summary, winter wheat growth had great effect on soil NN content, whereas little effect on TN and AK. The differences of NN, TN and AK between June and April were negative in most areas and the absorptive capacity of winter wheat was different in different places.
\end{abstract}

Keywords: spatial - temporal variability, soil nutrients, geostatistics.

\section{Introduction}

Many researchers pointed that the variability of soil nutrients were affected by many factors, such as, land use types[1], soil types[2], planting types[3-8], topography[9-10], crop rotation[11-12] and so on. There were also some studies on the variability of soil nutrients variables in winter wheat field. Zhang et al[13] researched the spatial variability of soil properties including $\mathrm{TN}$, available $\mathrm{N}$, organic matter, available $\mathrm{P}, \mathrm{AK}$ and so on in winter wheat field in March 2002. Xie et al[14] analyzed the spatial-temporal variations of purple soil ammonia and $\mathrm{NN}$ under application of inorganic and organic fertilizers in winter wheat field. Chen et al[15] studied the law of spatial-temporal change of soil organic matter, total $\mathrm{N}$, available $\mathrm{N}$, available $\mathrm{P}$, and available K in farmland for last 20 years(1981 2000). However, little study studied the spatial and temporal changes of soil nutrients during winter wheat growth season.

\footnotetext{
* Corresponding authors.

D. Li and Y. Chen (Eds.): CCTA 2012, Part II, IFIP AICT 393, pp. 238-247, 2013.

(C) IFIP International Federation for Information Processing 2013
} 
This paper studied the spatial-temporal variability characteristics of soil NN, TN and AK during wheat growth period. The study tried to reveal the dynamic change rule of soil nutrients in winter wheat field, in order to prove theoretical basis for winter wheat precise management.

\section{Materials and Methods}

\subsection{Study Area}

The study were conducted at National Experiment Station for Precision Agriculture,

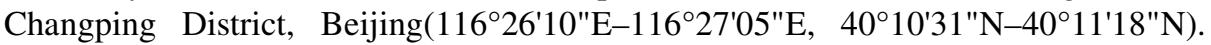
The experimental plot had an area of 5.4 ha (Figure 1), and soil type was alluvial soil. Winter wheat (Triticum aestivum L.) cultivar ('Jingdong-8') was selected in this study.

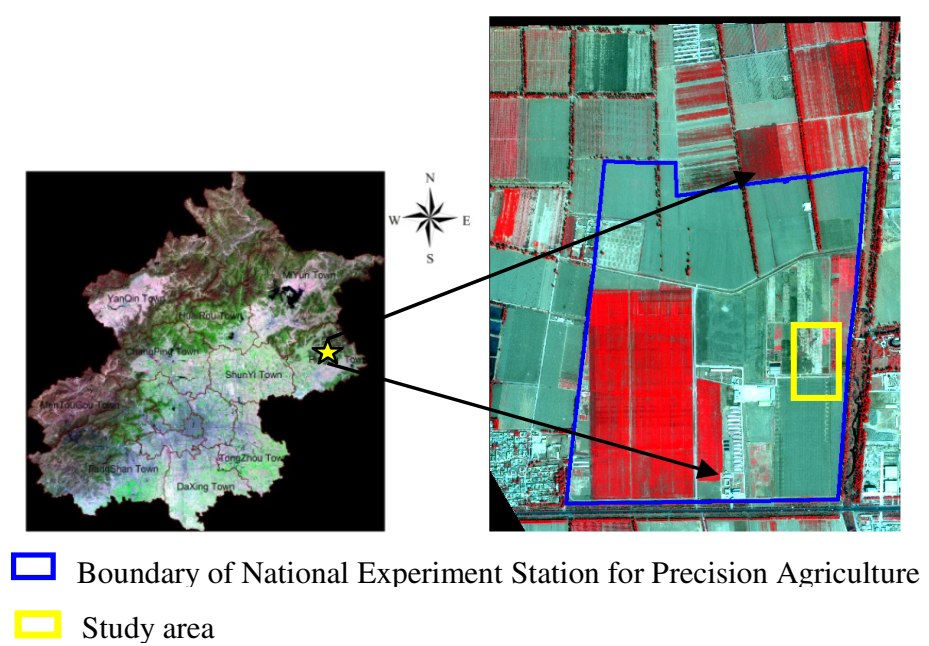

Fig. 1. Location of study area

\subsection{Soil Sampling and Laboratory Analysis}

Soil samples from 0 - to $30-\mathrm{cm}$ depth were collected within 51 locations on $30 \times 30 \mathrm{~m}$ grid and 81 locations on $10 \times 10 \mathrm{~m}$ grid (Figure 2). The first set of soil samples was taken on April 4, 2006 just before fertilizer application at the wheat elongation stage. The second set of soil samples was taken on June 16, 2006 just before the wheat harvest. The location of each soil sample was recorded by a GPS receiver with a station-based differential signal (DGPS, Trimble 5700 RTK), of which the measuring accuracy was controlled within $20 \mathrm{~cm}$. The soil samples were taken manually using a stainless steel probe. For each plot, a composite soil sample comprising five random samples from within a $5 \mathrm{~m}$ radius and a depth of $0 \sim 30 \mathrm{~cm}$ was taken, then placed into its designated 
plastic bags, mixed, and transport back to the laboratory. Soil samples were air dried, crushed, sieved, and analyzed for soil NN, TN and AK using the agro chemistry conventional analysis method[16].

\subsection{Analysis Methods}

In this study, classical statistical methods and geostatistics methods were used to analyze spatial - temporal variability of soil nutrients. Through the classical statistical methods, the minimum, maximum, mean, skewness, kurtosis, standard deviation and coefficient of variation were got. The $\mathrm{CV}$ of variables can be used to roughly estimate the variation degree: if the value of $\mathrm{CV}$ was $<10 \%$, the variable was considered weak variability; if the value was $>10 \%$ and $<100 \%$, the variable was considered the medium-degree variability; if the value was $>100 \%$, the variable was considered the strong variability[17].

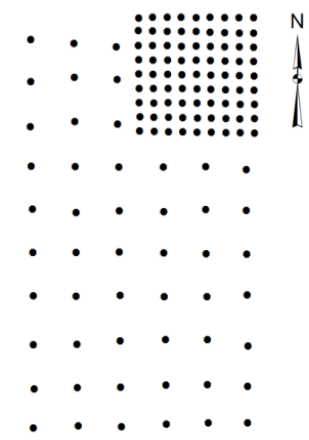

Fig. 2. The distribution of soil sampling points

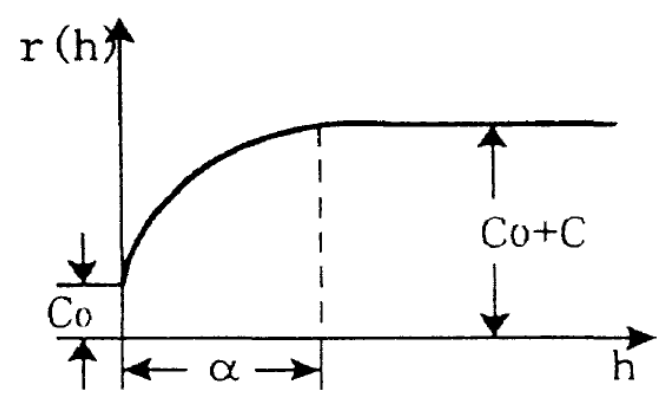

Fig. 3. Semivariogram variation curve

Figure 3 was a ideal semivariogram variation curve[18]. A semivariogram model consists of three important parameters which are $\mathrm{C}_{0}, \mathrm{C}+\mathrm{C}_{0}$ and range. $\mathrm{C}_{0}$ represents the nugget effect, which is the semivariogram value when the distance is zero. The nugget effect is led to random factors, such as sampling error and measurement error. $C+C_{0}$ is the sill, which represents total variance. We adopted the value of $\mathrm{C}_{0} /\left(\mathrm{C}+\mathrm{C}_{0}\right)$ to stand for the spatial class. If the ratio of spatial class was $<25 \%$, the variable was considered strongly spatially dependent ; If the ratio of spatial class was $>25 \%$ and $<75 \%$, the variable was considered moderate spatially dependent; and if the ratio was $>75 \%$, the variable was considered weakly spatially dependent[19]. Range is also known as the longest correlation distance. If samples separated by distances are smaller than the range, they are related spatially in some extent; if those separated by distances are greater than the range, there were no spatial dependence[20].

The classical statistical analysis of soil parameters were operated with SPSS11.5 software. Variowin 2.2[21] was used to compute the variograms of semivariogram and Arcgis9.0 was used for kriging interpolation. To characterize the spatial variation of the 
soil properties, experimental variograms were computed using Variowin 2.2. The parameters of the fitted models were then used with the data for ordinary kriging in Arcgis9.0. The values of the soil properties were predicted on grid at un-sampled locations and the predictions were used to produce interpolation maps.

\section{$3 \quad$ Results and Discussion}

\subsection{Simple Statistics of Soil Nutrients Data}

Average value and coefficients of variation for soil NN, TN and AK were listed in Table1. The average value of NN content $(19.20 \mathrm{mg} / \mathrm{kg})$ in April was greater than in June $(3.51 \mathrm{mg} / \mathrm{kg})$. The dramatic decrease was mainly caused by the NN absorption of winter wheat during the growth season of elongation stage to mature stage from April to June. Compared with the average value of AK in April $(137.60 \mathrm{mg} / \mathrm{kg})$, the mean value in June decreased slightly $(110.67 \mathrm{mg} / \mathrm{kg})$. But there were no change of TN content on average between in April and in June. In April, the variation coefficient (CV) of NN was $38.59 \%$, which was greater than AK (18.72\%) and TN (13.44\%). In June, CV of NN increased to $61.05 \%$. TN had the smallest CV of $12.36 \%$ in June. The $\mathrm{CV}$ of AK was $18.72 \%$ in April and $14.32 \%$ in June. All soil nutrients variables both in April and June were medium-degree variations with CV of $12.36 \% \sim 61.05 \%$.

Compared with CV of soil nutrients variables in April, CV for all soil nutrients in June changed more or less. Except NN with a marked increase of the CV from $38.59 \%$ to $61.05 \%$, the $\mathrm{CV}$ of other soil nutrients decreased in different degree from April to June.

Table 1. Descriptive statistical of soil nutrients properties in April and June

\begin{tabular}{llllllll}
\hline Variables & Minimum & Maximum & Mean & SD & CV\% & Skewness & Kurtosis \\
\hline $\begin{array}{l}\text { NN-April } \\
\text { (mg/kg) }\end{array}$ & 6.85 & 46.78 & 19.20 & 7.41 & 38.59 & 0.01 & -0.25 \\
TN -April & 0.08 & 0.13 & 0.10 & 0.01 & 13.44 & 0.32 & -0.62 \\
$\begin{array}{l}(\%) \\
\text { AK-April }\end{array}$ & 77.30 & 237.20 & 137.60 & 25.77 & 18.72 & -0.20 & 0.27 \\
$\begin{array}{l}\text { (mg/kg) } \\
\text { NN-June }\end{array}$ & 0.96 & 15.13 & 3.51 & 2.14 & 61.05 & 0.50 & 0.67 \\
$\begin{array}{l}\text { (mg/kg) } \\
\text { TN-June }\end{array}$ & 0.07 & 0.13 & 0.10 & 0.01 & 12.36 & 0.19 & -0.65 \\
$\begin{array}{l}\text { (\%) } \\
\text { AK-June }\end{array}$ & 80.55 & 171.11 & 110.67 & 15.84 & 14.32 & 0.25 & 0.02 \\
$(\mathrm{mg} / \mathrm{kg})$ & & & & & & & \\
\hline
\end{tabular}

Note: $\mathrm{NN}=$ nitrate nitrogen; $\mathrm{TN}=$ total nitrogen; $\mathrm{AK}=$ available potassium; $\mathrm{CV}=$ variation coefficient. 
Skewness and kurtosis values of soil variables indicated that only the soil TN in April and June was in normally distribution. The other soil properties were in nearly normal distribution after natural logarithm transformation before geostatistics analysis. Skewness and kurtosis values of all soil properties were also given in Table 1.

\subsection{Spatial Structure Analysis of Soil Nutrients in Winter Wheat Fields}

The semivariogram parameters of soil properties were shown in Table 2. The semivariogram of soil TN in June showed Spherical model, whereas other soil nutrients both in April or June showed Gaussian model.

Table 2. The semivariogram parameters of soil properties

\begin{tabular}{llllll}
\hline Variables & Model & Nugget $\left(\mathrm{C}_{0}\right)$ & Sill $\left(\mathrm{C}+\mathrm{C}_{0}\right)$ & Range $(\mathrm{m})$ & Spatial class \\
\hline NN-April & Gaussian & 0.109887 & 0.17788 & 170.00 & 61.78 \\
TN -April & Gaussian & 0.000090 & 0.00019 & 150.00 & 48.39 \\
AK-April & Gaussian & 0.018496 & 0.04910 & 190.00 & 37.67 \\
NN-June & Gaussian & 0.209590 & 0.27857 & 220.00 & 75.24 \\
TN -June & Spherical & 0.000040 & 0.00017 & 170.00 & 23.26 \\
AK-June & Gaussian & 0.007603 & 0.01780 & 61.19 & 42.71 \\
$\Delta$ NN & Exponential & 34.285300 & 52.41203 & 98.83 & 65.41 \\
$\Delta \mathrm{TN}$ & Gaussian & 0.000024 & 0.00004 & 19.30 & 66.67 \\
$\Delta \mathrm{AK}$ & Exponential & 270.252000 & 578.01600 & 93.59 & 46.76 \\
\hline
\end{tabular}

Note: $\mathrm{NN}=$ nitrate nitrogen; $\mathrm{TN}=$ total nitrogen; $\mathrm{AK}=$ available potassium; $\Delta \mathrm{NN}=$ nitrate nitrogen content in June minus in April; $\Delta \mathrm{TN}=$ total nitrogen content in June minus in April; $\Delta \mathrm{AK}=$ available potassium content in June minus in April.

The spatial class of TN in June (23.26\%) was smaller than 25\%, which indicated the existence of strong spatial dependence. The spatial class of NN in June was $75.24 \%$, more than $75 \%$. The other soil nutrients variables had moderate spatial dependence with the spatial class at the range of $37.67 \% \sim 61.78 \%$.

Except AK with a marked decrease of the range, the ranges of other soil nutrients increased about 20m from April to June. The range of AK in April was $220.00 \mathrm{~m}$, whereas that in June decreased to $61.19 \mathrm{~m}$. The ranges of all soil variables were bigger than the sampling interval of $30 \mathrm{~m}$, which satisfied the effectiveness of geo-statistical analysis. 
Table 3 presented the cross validation results of the optimal model for soil variables. Generally, the optimal model should be the one that Root-Mean-Square (RMS) is closer to Average Standardized Error (ASE), Mean Standardized (MS) is closer to 0, and Root-Mean-Square Standardized (RMSS) is closer to 1.

Table 3. The cross validation results of semivariogram model optimal model for each soil variable

\begin{tabular}{lllll}
\hline Variables & RMS & ASE & MS & RMSS \\
\hline NN-April & 6.661 & 7.334 & -0.016 & 0.937 \\
TN -April & 0.009 & 0.010 & 0.004 & 0.911 \\
AK-April & 20.900 & 20.320 & -0.010 & 1.043 \\
NN-June & 2.141 & 1.845 & -0.063 & 1.193 \\
TN -June & 0.007 & 0.008 & 0.004 & 0.870 \\
AK-June & 11.240 & 11.630 & -0.007 & 0.968 \\
$\Delta$ NN & 6.163 & 6.730 & 0.012 & 0.925 \\
$\Delta \mathrm{TN}$ & 0.006 & 0.006 & -0.001 & 0.968 \\
$\Delta \mathrm{AK}$ & 21.240 & 20.890 & 0.008 & 1.020 \\
\hline
\end{tabular}

Note: $\mathrm{NN}=$ nitrate nitrogen; $\mathrm{TN}$ = total nitrogen; $\mathrm{AK}$ = available potassium; RMS = Root-Mean-Square, ASE $=$ Average Standardized Error, MS $=$ Mean Standardized, RMSS $=$ Root-Mean-Square Standardized .

\subsection{The Spatial Distribution Maps of Soil Nutrients in Winter Wheat Field}

Figure 4 indicated that, soils with high NN content in April distributed in the north and east part of the field with downward trend from SW to EN. NN content in most areas of study area was lower than the average value $(19.20 \mathrm{mg} / \mathrm{kg})$. Different to the map of NN in April, high NN content in June were found in the north part of the field, whereas, low NN content located in the east part of the field. The NN content average value in June was lower than that of in April $(19.20 \mathrm{mg} / \mathrm{kg})$. It indicated that winter wheat growth strongly influenced the soil NN content and spatial distribution.

The spatial distribution map of TN in April showed that soil with high TN content was distributed in the northeastern of field and it reduced from SW to EN, which was similar to the distribution of TN in June. This implied that the spatial distribution of soil TN had low change during winter wheat growth.

Soil with high AK content in April was located in the north part of the field, and AK content in most areas was lower than the average value $(137.60 \mathrm{mg} / \mathrm{kg})$. In June, the soil distributed in the north and east part of field had high AK content, and the content in most areas was lower than the average value of $110.67 \mathrm{mg} / \mathrm{kg}$. The soil AK of research field both April and June had the similar spatial distribution with high content in the EN part and low in the SW part. 

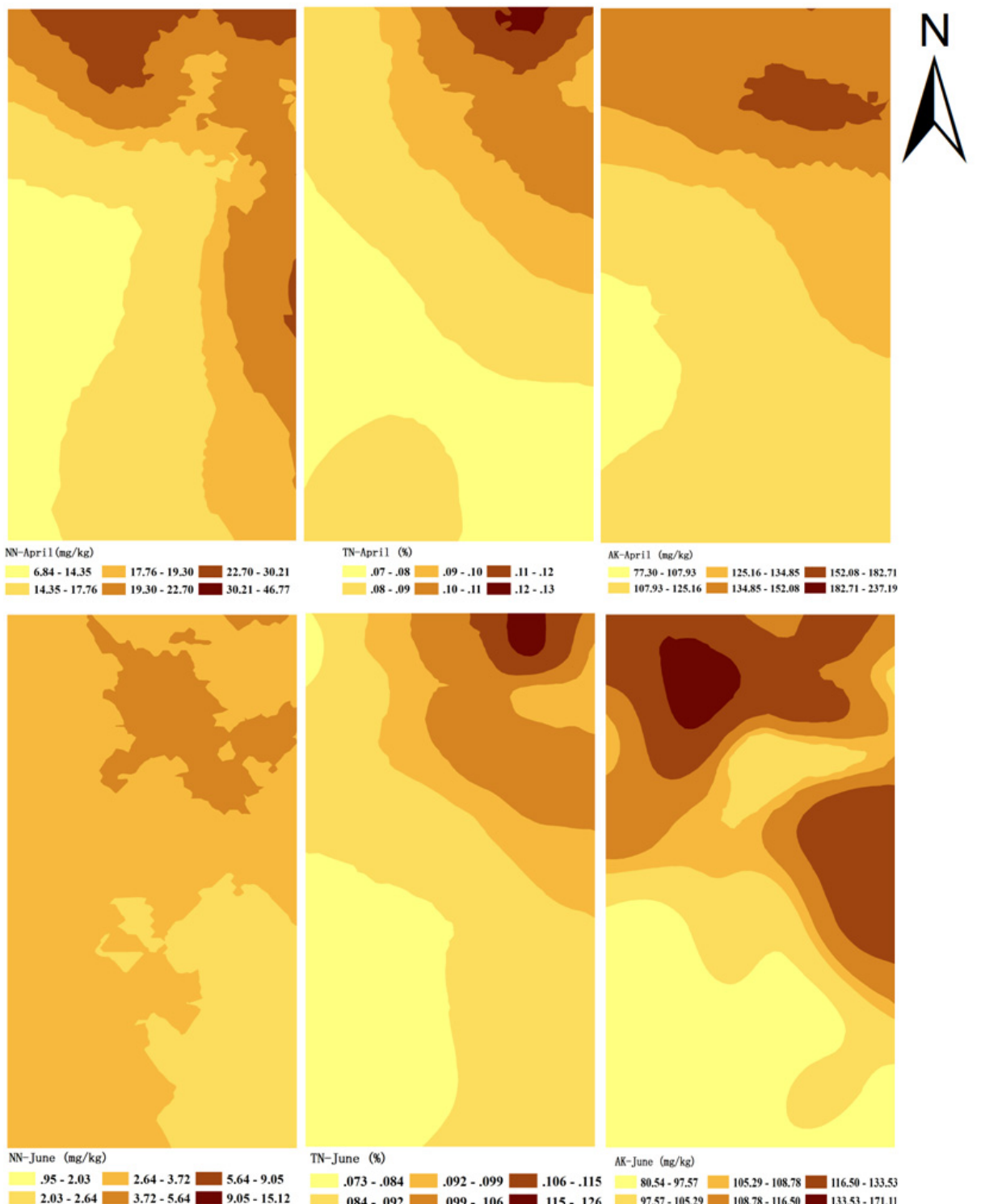

TN-June (\%)
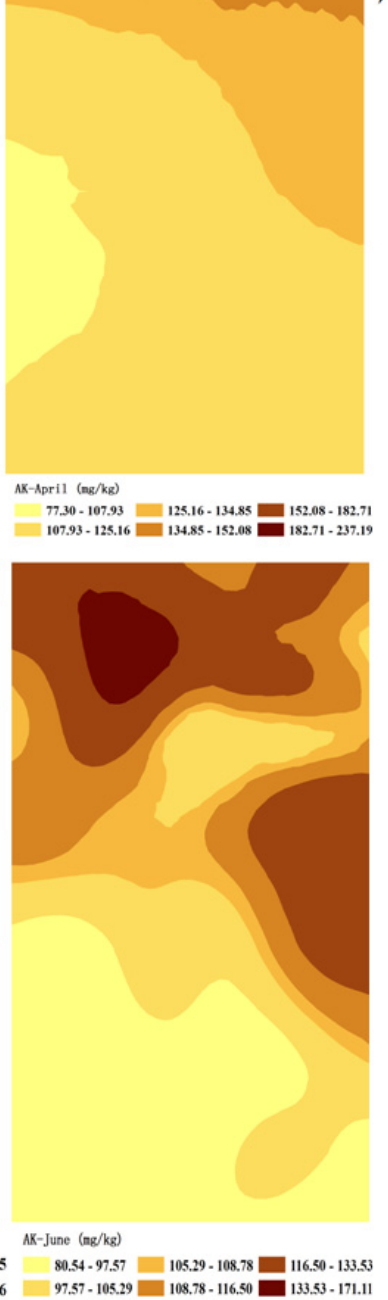

Fig. 4. The spatial distribution maps of nitrate nitrogen (NN), total nitrogen (TN) and available potassium (AK) in April and June 


\subsection{Temporal Variability Analysis of Soil Nutrients in Winter Wheat Field}

In order to clearly reflect the change of soil nutrients during winter wheat growth season, the differences spatial distribution of soil NN, TN and AK between April and June were calculated using the band match function of ENVI software.

Figure 5 showed the differences spatial distribution of NN, TN and AK between April and June. From those, the differences of NN, TN and AK were negative in most areas, which indicated winter wheat not only absorbed original fertilizer but also absorbed the topdressing at the wheat elongation stage. In different places, the changes of nutrients were not same, which indicated the absorptive capacity of winter wheat on soil nutrients were different in this field.
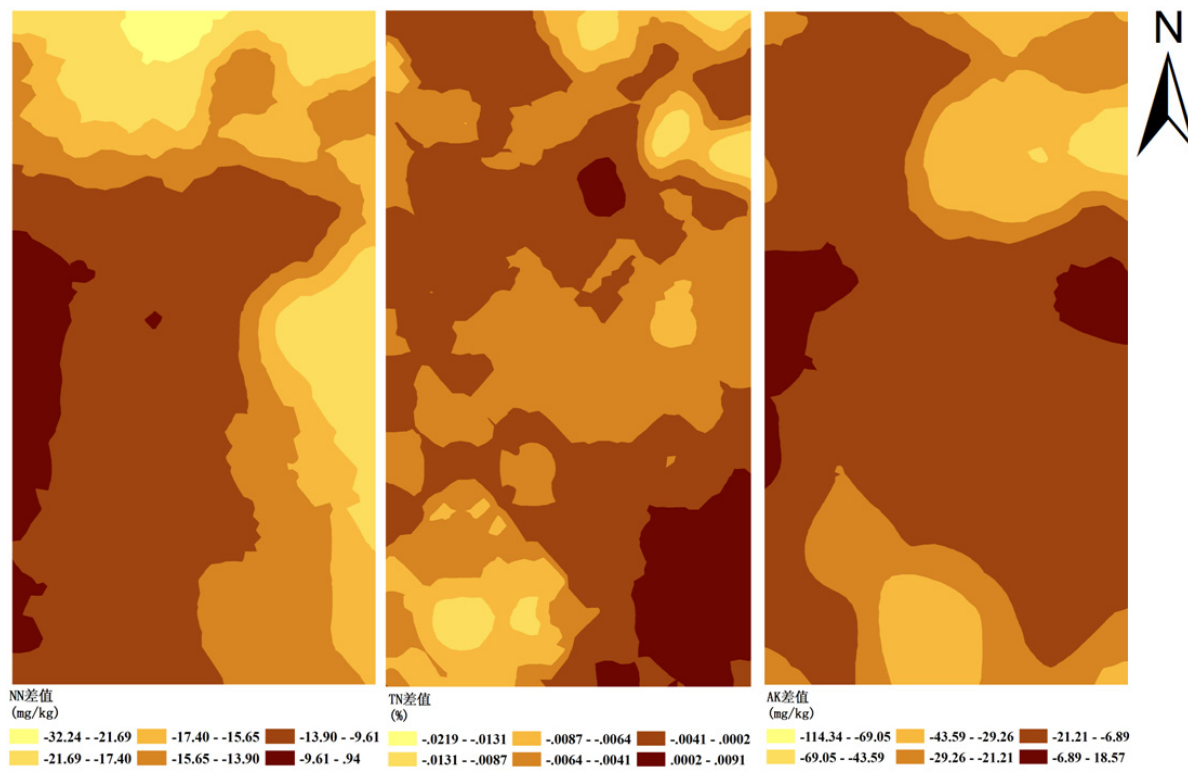

Fig. 5. The spatial distribution maps of differences of nitrate nitrogen $(\mathrm{NN})$, total nitrogen (TN) and available potassium (AK) between April and June

\section{Conclusions}

Compared with the average value of $\mathrm{NN}$ and $\mathrm{AK}$, the average value of $\mathrm{NN}$ content decreased obviously in June, and the value of AK in June decreased slightly. But there were no change of TN content on average between April and June. All soil variables both in April and June were medium-degree variations with CV of $12.36 \% \sim 61.05 \%$.

From the spatial distribution maps of soil nutrient, the NN changed obviously on spatial distribution between April and June. However, the spatial distribution of soil TN and AK changed small, which had the similar spatial distribution that high content in 
the northeast part and low in the southwest part. Winter wheat growth had great effect on soil NN, whereas little effect on TN and AK.

The differences of NN, TN and AK between June and April were negative in most areas, and the changes of nutrients were not same in different places. These indicated that winter wheat growth need large amount of nitrogen and potassium fertilizer and different absorptive capacity of winter wheat on soil nutrients in different places.

Acknowledgements. This work was financially supported by program of National Natural Science Foundation of China (31071324), program of National Natural Science Foundation of China (41201326) and Shanxi Province Science and Technology Support Program (20110311038), program of State Key Basic Research and Development Program (2011CB311806).

\section{References}

1. Qin, J., Kong, X.B., Jiang, G.H., Liu, Y., Li, C.Z.: Characteristics of Spatio-Temporal Changes of Soil Organic Matter in Typical Fringe in Beijing for 25 Years. Transactions of the CSAE 24(3), 124-129 (2008)

2. Zhao, Y.F., Shi, X.Z., Yu, D.S., Huang, B., Wang, H.J., Zhao, Y.C., et al.: Spatial variation of soil nutrients and its affecting factors at small scale - A case study of peri-urban areas in Wuxi Jiangsu Province. Chinese Journal of Soil Science 37(2), 215-219 (2006)

3. Zhang, Q.J., Jiang, H.L., Liu, G.S., Wang, X.J., Liu, Y.Q., Liu, Q.H., Gu, J.G., Liu, H.W.: Research on Spatial Variability of Soil Nutrients in Tobacco Plantation Field. Journal of Henan Agricultural University 45(1), 116-122 (2011)

4. Qi, Z.P., Wei, Z.Y., Li, F.Y., Tang, S.M.: Spatial-temporal Variability of Paddy Soil Nutrient in Hainan Province. Chinese Journal of Soil Science 40(6), 1292-1296 (2009)

5. Peng, T., Lv, X.: Study on Spatio-Temporal Variation of Soil Organic Matter in Oasis Cotton Field. Chinese Journal of Soil Science 41(3), 563-567 (2010)

6. Wang, B., Long, H.Y., ZhuGe, Y.P., Zhang, J.G., Gong, Y.K.: Study on Spatio-Temporal Variability of Soil Available Nutrients in Typical Vegetable Field of South China: A Case Study at Guangxingzhou, Junshan, Yueyang. Plant Nutrient and Fertilizer Science 15(3), 551-558 (2009)

7. Liu, Q.F., Lu, J.L., Li, S.L., Sheng, H.Y., Li, N., Wang, Y.P., Wang, J.M., Zhu, C.L.: The Spatio-Temporal Variation of Nitric Nitrogen in Protected Vegetable Soils in Different Years of Cultivation. Agricultural Research in the Arid Areas 29(2), 159-163 (2011)

8. Huang, Z.G., Li, B.G., Hu, K.L.: Characteristics of the Spatio-Temporal Changes of Soil Organic Matter of Sugarcane Field in Red Soil Hill Areas. Transactions of the CSAE 22(11), 58-63 (2006)

9. Umali, B.P., Oliver, D.P., Forrester, S., Chittleborough, D.J., Hutson, J.L., Kookana, R.S., Ostendorf, B.: The Effect of Terrain and Management on the Spatial Variability of Soil Properties in an Apple Orchard. Catena 93, 38-48 (2012)

10. Hattar, B.I., Taimeh, A.Y., Ziadat, F.M.: Variation in Soil Chemical Properties along Toposequences in an Arid Region of the Levant. Catena 83(1), 34-45 (2010)

11. Huang, B., Sun, W.X., Zhao, Y.C., Zhu, J., Yang, R.Q., Zou, Z., Ding, F., Su, J.P.: Temporal and Spatial Variability of Soil Organic Matter and Total Nitrogen in an Agricultural Ecosystem as Affected by Farming Practices. Geoderma 139, 336-345 (2007) 
12. Song, G., Sun, B.: Spatial and Temporal Changes of Soil Inorganic Nitrogen in Rice-wheat Rotation System at County Scale-A Case Study in Yizheng County, Jiangsu Province, China. Journal of Agro-Environment Science 28(2), 636-642 (2008)

13. Zhang, S.J., He, Y., Fang, H.: Spatial Variability of Soil Properties in the Field Based on GPS and GIS. Transactions of the CSAE 19(2), 39-44 (2003)

14. Xie, H.M., Zhu, B., Zhu, Z.L.: Temporal-Spatial Variations of Ammonia and Nitrate under Application of Inorganic and Organic Fertilizers in Purple Soil of Winter Wheat. CJEA 14(1), 118-121 (2006)

15. Chen, F., Pu, L.J., Cao, H., Peng, B.Z., Yang, G.S., Zhou, S.L.: Spatial and Temporal Changes for Soil Nutrients and Their Mechanism in Typical Area of Taihu Lake Valley During the Past Two Decades. Acta Pedologica Sinica 39(2), 236-245 (2002)

16. Nanjing Agriculture College. Analysis Method of Agricultural Soil, pp. 25-69. Agriculture Press, Beijing (1980)

17. Wang, S.Q., Zhu, S.L., Zhou, C.H.: Characteristics of Spatial Variability of Soil Thickness in China. Geographical Research 20(2), 161-169 (2001)

18. Chen, L.P.: Theoretical and Experimental Studies on Variable-Rate Fertilization in Precision Farming. China Agricultural University, Beijing (2003)

19. Iqbal, J., Thomasson, J.A., Jenkins, J.N., Owens, P.R., Whisler, F.D.: Spatial Variability Analysis of Soil Physical Properties of Alluvial Soils. Soil Sci. Soc. Am. J. 69, 1338-1350 (2005)

20. Cambardella, C.A., Moorman, T.B., Novak, J.M., Parkin, T.B., Karlen, D.L., Turco, R.F., Konpka, A.E.: Field-Scale Variability of Soil Properties in Central Iowa Soils. Soil Sci. Am. J. 58, 1501-1511 (1994)

21. Pannatier, Y.: Variowin Software for Spatial Data Analysis in 2D. Springer, New York (1996) 